\title{
A Comparison of Need within a Social Business Model: Residentially Stable and Residentially Unstable Substance Abusers
}

\author{
John Conahan, Don MacIntyre \\ Social Work Department, Kutztown University, Kutztown, USA \\ Email: conahan@kutztown.edu, don@gatehouse.org
}

Received 23 July 2015; accepted 12 September 2015; published 15 September 2015

Copyright (C) 2015 by authors and Scientific Research Publishing Inc.

This work is licensed under the Creative Commons Attribution International License (CC BY). http://creativecommons.org/licenses/by/4.0/

(c) () Open Access

\section{Abstract}

This study is a mixed design including qualitative and quantitative data gathering for both descriptive and exploratory purposes. The fundamental research question is how do the recovery needs of residentially unstable substance abusers who are provided with transitional housing within a social business model compare with the recovery needs of substance abusers who are not residentially unstable.

\section{Keywords}

\section{Substance Abuse, Homelessness, Residential Instability, Social Business}

\section{Introduction}

Homelessness and other forms of residential instability are emerging as a primary recovery issue among substance abusing populations and represent an important threat to the recovery process of those discharged from in-patient treatment (Conahan \& MacIntyre, 2012 [1]; Callaghan \& Cunningham, 2002 [2]; Moos, Nichol, \& Moos, 2002) [3].

The focus organization of this study (the "Halfway House") is a drug and alcohol rehabilitation organization operating at level-of-care 2B ("Halfway House") as defined by the Common Wealth of Pennsylvania, Department of Health, Bureau of Drug and Alcohol Programs, Client Placement Criteria (1999) [4]. Clients at this level of care must have had previous in-patient drug and alcohol treatment and still require additional inpatient care in a structured living environment. The usual length of stay is 90 days. Treatment plans are individualized and gender specific, and services include individual, group, and family counseling; 12-step facilitation; psychiatric evaluations; co-occurring mental disorder treatment; relapse prevention training; spiritual counseling; life skills 
training including vocational readiness, budgeting, communications skills, and parenting classes; and aftercare planning. Virtually all clients are publicly funded $(99 \%+$ for the last 6 months). Of the subjects of the current study, about $70 \%$ have a co-occurring mental disorder in addition to their substance use disorder.

For the fiscal year for which clients were selected for this study, $78 \%$ were residentially unstable at the time of their discharge, meaning that they either had no home to return to or could only return to home that was inconsistent with their continuing recovery from addiction. As described below, homelessness and other forms of residential instability are a significant threat to the recovery of many addicts. In order to address this problem, this program developed and implemented a social business model plan for an eight bed men's transitional home for graduates of their three-month program who were in need of housing, with the first clients moving in September 2009 (Conahan \& MacIntyre, 2012) [1]. (Transitional living homes for addicts are also called "recovery houses" and are an unregulated level of care in Pennsylvania at the time of this study.)

The purpose of this study was to investigate whether men referred to the transitional living facility upon graduation from the 90-day program due to their residential instability, benefited from this opportunity by comparing their recovery on both quantitative and qualitative measures with graduates of the Halfway House who were not residentially unstable and therefore were not referred to the transitional living facility.

\section{Literature Review}

Addiction is a chronic disease (O’Brien \& McLellan, 1996, p. 238) [5] and recovery is a long-term process. For example, White and Kurtz (2006) [6] have found that "symptoms continue to improve or remit over the first ten years of recovery" (p. 13). Despite this fact, drug and alcohol service organizations continue to provide acute treatment, i.e. short periods of intensive care, after which patients are discharged and often left to their own resources.

Thus, a gap exists between a chronic disease and acute treatment, and there are many threats to client sobriety upon discharge. Among these, homelessness and other forms of residential instability are particularly menacing and much research exists demonstrating a strong relationship between substance abuse and homelessness (McKeown, 2007 [7]; Anderson, Shannon, Schyb, \& Goldstein, 2002 [8]; Devine \& Wright, 1997 [9]; Stahler \& Cohen, 1995 [10]). Also, residential instability and homelessness among addicts are related to a variety of negative recovery outcomes. For example, Callaghan and Cunningham (2002) [2] reveal that "the lack of a fixed address" (p. 405) is a risk factor for re-admission to detoxification. Residential instability, defined as "1 year or less in the same setting” (Moos et al., 2002, p. 78) [3], is a risk factor for increased drug use symptoms after discharge from in-patient treatment, and homeless addicts achieve better abstinence outcomes when they are provided with housing (Milby, Schumacher, Wallace, Freedman, \& Vuchinich, 2005) [11].

For purposes of recovery from addiction, residential instability for substance abusers must also include homes that provide a significant threat to sobriety because drug or alcohol use occurs there. According to Bond, Kaskutas, and Weisner (2003) [12], the risk of relapse in the year following in-patient treatment increases in proportion to the number of heavy or problem drinkers in an alcoholic's post-treatment social network, and Salomonsen-Sautel et al. (2008) [13] note that "homeless youths" (p. 230) suffer a high risk of having parents with substance use problems.

\section{Treatment Organization Response}

Due to the very limited body of research in this area, the need exists to study how one organization addressed this issue in order to build a conceptual model for future study as well as clarify organizational strategies and protocols to address homelessness in the substance abusing population. The organizational response and decision making process to address the housing need was grounded in the social business model asserted by Yunus (2010) [14].

Yunus, (2010) [14] in a response to address the social and economic injustice of poverty, proposed the concept of a social business model to address and eliminate poverty. A social business model is a cause-driven business. The purpose of an investment in a social business is to achieve one or more social business objectives - no personal gain is desired by the organizational leadership or ownership. Yunus (2010) [14] describes two types of social business models. The first is the business dealing with social objectives only, with the product produced for the benefit of the clients or customers. The second model is any business that allows the poor to own the business in order to gain through direct dividends. The organization in this study, guided by it mission 
and organizational values, invested in the social objective of housing for individuals suffering from a substance use disorder.

The treatment organization in this study is a drug and alcohol rehabilitation organization with two facilities (men and women) in existence for 35 years in the Northeastern United States. Over the period from mid-2005, when discussions began, to September 2009, when the first clients moved in, the treatment organization developed and implemented a plan for an eight bed transitional home for graduates of their three-month residential treatment program who were in need of housing. The development of this transitional home reflected a perception by this organization that the need for housing was an important issue in the recovery of many of their graduates. The organization in this study is driven by the mission "To provide effective gender-specific behavioral health services that assist chemically dependent people establishing lives in recovery" (organizational strategic plan, 2007). The vision and values of this organization promote a full continuum of care, when indicated, with comprehensive services.

The authors believe that the literature demonstrates a great need for housing among residentially unstable sufferers of a substance use disorder. However, what separates this case from others is the organization's congruency with the social business model described by Yunus (2010) [14].

The social business model is defined by two types; type one focuses on businesses, not-for-profit or for-profit, that deal with social objectives only. The type two model is identified by ownership by the poor or in this case, the homeless recovering substance abusers. This case study suggests that this particular organization adopted its business approach with the social objective of resolving the lack of suitable, safe and affordable transitional housing as its primary focus. The data obtained from committee minutes, finance meetings and the organization's decision to create a separate organization and donate the funds to purchase the home, rather than loan them, serves as support for this type of social business model (Conahan \& MacIntyre, 2012) [1].

\section{Study Purpose}

This study is a mixed design including qualitative and quantitative data gathering for both descriptive and exploratory purposes. This investigation is a follow up and a next step in investigating the implementation and outcome of a social business model designed to address residentially unstable substance abusers with transitional housing services. The fundamental research question is "how do the recovery needs of residentially unstable substance abusers who are provided with transitional housing compare with the recovery needs of substance abusers who are not residentially unstable?"

To determine if clients sent to the transitional home benefitted from this opportunity with respect to their recovery, with "benefitted" to be defined broadly based on whether or not they were as likely as comparable clients who completed treatment at the Halfway House and did not need transitional living services, to complete their follow-up treatment plan, gain/maintain employment, find supportive living arrangements, attend AA/NA meeting, and to need additional social services.

\section{Method}

Every graduate who went to the transitional living home upon graduation was invited to participate in this study. Those who agreed to participate were matched with a Halfway House graduate who did not need a transitional living arrangement because they had a stable home to return to, in order to create the comparison group. This matching was based on the bio-psychosocial assessment done by the Halfway House at admission to halfway house treatment and considered criteria including substance of abuse, co-occurring mental disorders, prior treatment, time since graduation, age, employment status, legal involvement, ethnicity, biopsychosocial needs upon admission and discharge, and medications taken. Initially the instrument used to compare these two groups was a version of the Addiction Severity Index (ASI), called the ASI-Lite (McLellan, Cacciola, Alterman, Rikoon, \& Carise, 2006) [15]. This instrument was selected because it is a commonly used measure of the construct of addiction severity, has know psychometric properties, is in the public domain, requires no interviewer ratings (unlike the full ASI), and measures substance abuser lives across seven different dimension considered to be relevant to this research question. However, due to difficulties contacting Halfway House graduates after discharge, the authors utilized a closed record review to gather the appropriate information (Rubin \& Babie, 2008) [16].

For qualitative data, a convenience sample of members of both the intervention and comparison groups was 
gathered for a professionally facilitated focus group to explore issues around their addictions, their recoveries, and how both are affected by their housing situations. In order to add greater depth to this investigation, direct service counselors and clinical supervisors were also interviewed. A discussion guide was used to stimulate discussion and covered topics including client's perceptions of services provided and factors affecting recovery (including housing) in order to supplement, refine, and augment quantitative findings.

\section{Data Collection}

To determine if clients sent to the transitional home benefitted from this opportunity with respect to their recovery, with "benefitted" to be defined broadly based on whether or not they were as likely as comparable clients who completed treatment at the Halfway House and did not need transitional living services, to complete their follow-up treatment plan, gain/maintain employment, find supportive living arrangements, attend AA/NA meeting, and to need additional social services. The comparison groups consisted of two groups between which to do quantitative comparisons:

Group one $\mathrm{N}=20$ is composed of Halfway House graduates who were sent to transitional housing services because of their housing need (regardless of whether they completed the transitional service from or not).

Group two $\mathrm{N}=20$. Halfway House graduates who went home to a stable/supportive environment, preferably selected from among those who graduated from the Halfway House while the Transitional House was open.

A retrospective review and a convenient sample of closed records were utilized. The closed records selected were matched with a Halfway House graduate who did not need a transitional living arrangement because they had a stable home to return to, in order to create the comparison group. This matching was based on the biopsychosocial assessment done at admission to halfway house treatment and considered criteria including substance of abuse, co-occurring mental disorders, prior treatment, time since graduation, age, employment status, legal involvement, ethnicity, biopsychosocial needs upon admission and discharge, and medications taken.

For qualitative data collection, a convenience sample composed of selected members of both the intervention group and the comparison group was gathered in a focus group and their discussion recorded and transcribed. The resulting transcripts were analyzed as described below. Two researchers independently made lists of themes for the responses of each of the questions. One of the study authors, a social work graduate student, combined these lists based on overlapping themes to create a set of codes. Two study authors coded the responses. Responses to the open-ended questions (Appendix) comprised the focus of our qualitative analysis. The inter-rater reliability utilized was measured as 80 between the raters. Upon completion of the interviews, utilizing Appendix, the raters reviewed each other's independent coded responses and themes and counted the number of times agreement was reached. Since the score was well above 70 the basic benchmark for reliability it was conclude that a high degree of interrater reliability was reached (Rubin \& Babbie, 2008) [16].

\section{Data Analysis}

The output from the closed record review is a raw number and percentage for each of the domains this scale measures-medical status, employment/support status, substance use, drug use, legal status, family/social relations, and psychiatric status and medication. These scores will be compared between the intervention group and the comparison group to determine if the average composite scores for the two groups are significantly different. To the extent that they are different, these differences can, within the considerable constraints described below, be attributed, at least in part, to the difference in housing status of the two groups.

To evaluate qualitative data, the authors separately identified concepts in the focus group discussion and coded these. Then jointly, codes were agreed on and collapsed into themes. These themes were triangulated with the quantitative data yielded from the closed record review. "Triangulation is a validity procedure... and... systematic process of sorting through the data to find common themes or categories by eliminating overlapping areas” (Creswell and Miller, 2000, p. 127) [17]. Peer debriefing between the two authors was also used to establish trustworthiness and credibility. Peer debriefing "occurs when teams of investigators meet regularly to give each other feedback, emotional support, and ideas” (Rubin \& Babbie, 2008, p. 432) [16].

Between the qualitative themes that are revealed and the quantitative data concerning differences between residentially stable and unstable addicts, the authors intended to come to a better understanding of how residential instability affects addiction and recovery. To the extent that this effort is successful, such an understanding may raise overall awareness of this problem and offer insight into potential ameliorative interventions. 


\section{Strengths and Limitations}

A strength of this design is that including both quantitative and qualitative measures will allow the authors to look both deep and wide, seeking insights about the relationship between the biopsychosocial dimensions of residentially unstable residents who are provided with transitional housing and residents who are residentially stable, as well as exploring through a focus group of members of both groups, their experiences relative to housing and their addictions. It is hoped that this focus group will highlight issues and experiences not easily amenable to quantitative measures.

A potential weakness of this design is that the response rate of potential interviewees may be quite low, possibly subjecting our results to selection bias. If, for example, clients who are now homeless are less likely to participate because we have no way to reach them, then we would not know if those who chose to participate in our intervention group were representative of all clients who went to transitional services. A low response rate may also yield a small sample size, making statistical inference difficult or impossible.

A general weakness of posttest only designs is they are subject to "selection biases" (Rubin \& Babbie, 2008, p. 264) [16] in that one does not know whether any differences between the two groups at posttest are attributable to the "intervention" or were there to begin with. The current study tries to control for this weakness by matching clients in the two groups as described above, hopefully reducing any difference between them on dimensions considered critical.

\section{Results}

Table 1 displays demographics of age, race, and marital status. The data reveal no trends in demographic differences between residentially unstable and residentially stable groups. However, a high proportion of both groups have never been married.

Table 2 suggests that Heroin IV and Alcohol are the most common substances identified as problematic upon admission for the residentially unstable population and Cocaine and Opioid Non-IV being the third most utilized substance. The residentially stable population describes Alcohol, Heroin IV and cocaine as the most common substances of abuse.

Table 3 indicates that both groups identify multiple biopsychosocial needs upon admission. A third of the population from both groups identifies multiple needs of unemployment, legal, housing and medical as the most consistently reported.

Table 1. Characteristics by program type.

\begin{tabular}{|c|c|c|c|c|c|c|}
\hline \multirow{2}{*}{ Characteristics } & \multicolumn{2}{|r|}{ RU } & \multicolumn{2}{|r|}{ RS } & \multicolumn{2}{|r|}{ Total } \\
\hline & $\mathrm{N}$ & Proportion (\%) & $\mathrm{N}$ & Proportion (\%) & $\mathrm{N}$ & Proportion (\%) \\
\hline \multicolumn{7}{|l|}{ Age } \\
\hline $18-27$ & 8 & 44.4 & 5 & 25 & 13 & 32.5 \\
\hline $28-35$ & 5 & 25 & 7 & 35.3 & 13 & 32.5 \\
\hline $36-45$ & 5 & 25 & 7 & 35.3 & 12 & 30.8 \\
\hline 45 up & 2 & 0 & 2 & 10 & 2 & 5.1 \\
\hline \multicolumn{7}{|l|}{ Race } \\
\hline African American & 0 & 0 & 4 & 20 & 4 & 10.0 \\
\hline Caucasian & 17 & 85 & 14 & 73.7 & 31 & 79.5 \\
\hline Hispanic & 3 & 15 & 2 & 10.5 & 5 & 12.5 \\
\hline \multicolumn{7}{|l|}{ Marital status } \\
\hline Divorced & 2 & 10 & 5 & 25.1 & 7 & 17.5 \\
\hline Never married & 18 & 90 & 15 & 75 & 33 & 82.5 \\
\hline
\end{tabular}

Note: RU = resident; RS = residentially stable. 
Table 4 reveals the needs identified upon discharge. Both groups identified dual diagnosis needs upon discharge with the residentially stable group being slightly higher with $20.5 \%$. Housing needs, skilled training, medications and legal issues are a need for both groups.

In Table 5 dual diagnoses upon discharge were consistently reported by both groups with no difference between the groups. The most consistently identified dual diagnoses are depression, anxiety and bipolar disorder. The need for medication management was higher in the residential stable group at $38.5 \%$ and $30.8 \%$ for the residentially unstable population.

Table 2. Characteristics of use upon admission by program.

\begin{tabular}{|c|c|c|c|c|c|c|c|c|c|c|}
\hline \multirow{2}{*}{ Drugs upon admission } & \multicolumn{5}{|c|}{ RU } & \multicolumn{5}{|c|}{ RS } \\
\hline & $\mathrm{N}$ & Yes & $\%$ of Yes & $\mathrm{N}$ & $\%$ of No & $\mathrm{N}$ & $\mathrm{Y}$ & $\%$ of Yes & $\mathrm{N}$ & $\%$ of No \\
\hline Alcohol & 20 & 7 & 35.0 & 13 & 65.0 & 19 & 10 & 52.6 & 9 & 47.4 \\
\hline Heroin IV & 20 & 11 & 55.0 & 9 & 45.0 & 19 & 9 & 47.4 & 10 & 52.6 \\
\hline Polysub & 20 & 0 & 0.0 & 20 & 100.0 & 19 & 4 & 21.1 & 15 & 78.9 \\
\hline Cocaine & 20 & 6 & 30.0 & 14 & 70.0 & 19 & 9 & 47.4 & 10 & 52.6 \\
\hline Op noniv & 20 & 6 & 30.0 & 14 & 70.0 & 19 & 1 & 5.3 & 18 & 94.7 \\
\hline THC & 20 & 3 & 15.0 & 17 & 85.0 & 19 & 2 & 10.5 & 17 & 89.5 \\
\hline Benzo. & 20 & 3 & 15.0 & 17 & 85.0 & 19 & 0 & 0 & 19 & 100.0 \\
\hline Methamph & 20 & 1 & 5.0 & 19 & 95.0 & 19 & 0 & 0 & 19 & 100.0 \\
\hline Methadone & 20 & 1 & 5.0 & 19 & 95.0 & 19 & 0 & 0 & 19 & 100.0 \\
\hline PCP & 20 & 1 & 5.0 & 19 & 95.0 & 19 & 0 & 0 & 19 & 100.0 \\
\hline
\end{tabular}

Table 3. Characteristics of biopsychosocial needs upon admission by program.

\begin{tabular}{|cccccccccccc}
\hline Characteristics & \multicolumn{3}{c}{ RU } & \multicolumn{1}{c}{ RS } \\
\hline Biopsychosocial & $\mathrm{N}$ & Yes & \% of Yes & $\mathrm{N}$ & \% of No & $\mathrm{N}$ & $\mathrm{Y}$ & \% of Yes & $\mathrm{N}$ & $\%$ of No \\
\hline Multiple needs on admission & 20 & 7 & 35.0 & 13 & 65.0 & 19 & 7 & 36.8 & 12 & 63.2 \\
Legal & 20 & 15 & 75.0 & 5 & 25.0 & 19 & 12 & 63.2 & 7 & 36.8 \\
Housing & 20 & 6 & 30.0 & 14 & 70.0 & 19 & 5 & 26.3 & 14 & 73.7 \\
Existing family support & 20 & 6 & 30.0 & 14 & 70.0 & 19 & 6 & 31.6 & 13 & 68.4 \\
Employed & 20 & 10 & 50.0 & 10 & 50.0 & 19 & 11 & 57.9 & 8 & 42.1 \\
Medical issues & 20 & 10 & 50.0 & 10 & 50.0 & 19 & 11 & 57.9 & 8 & 42.1 \\
\hline
\end{tabular}

Table 4. Characteristics of discharge priorities upon admission by program.

\begin{tabular}{cccccccccccc}
\hline Characteristics & \multicolumn{9}{c}{ RU } & \multicolumn{1}{c}{ RS } \\
\hline Biopsychosocial & $\mathrm{N}$ & Yes & \% of Yes & $\mathrm{N}$ & \% of No & $\mathrm{N}$ & $\mathrm{Y}$ & \% of Yes & $\mathrm{N}$ & $\%$ of No \\
\hline Dual diagnosis & 20 & 6 & 30.0 & 14 & 70.0 & 19 & 8 & 42.1 & 11 & 57.9 \\
Mental health & 20 & 0 & 0.0 & 20 & 100.0 & 19 & 7 & 36.8 & 12 & 63.2 \\
Family support & 20 & 0 & 0.0 & 20 & 100.0 & 19 & 2 & 10.5 & 17 & 89.5 \\
Social support & 20 & 0 & 0.0 & 20 & 0.0 & 19 & 1 & 5.3 & 18 & 94.7 \\
Skilled training & 20 & 6 & 30.0 & 14 & 70.0 & 19 & 2 & 10.5 & 17 & 89.5 \\
Employed & 20 & 1 & 5.0 & 19 & 95.0 & 19 & 3 & 15.8 & 17 & 89.5 \\
Legal issues & 20 & 4 & 20.0 & 16 & 80.0 & 19 & 5 & 26.3 & 14 & 73.7 \\
Housing services & 20 & 14 & 70.0 & 6 & 30.0 & 19 & 12 & 63.2 & 7 & 36.8 \\
Medications & 20 & 5 & 25.0 & 15 & 75.0 & 19 & 9 & 47.4 & 10 & 52.6 \\
\hline
\end{tabular}


Table 5. Characteristics of dual diagnosis needs upon discharge by program.

\begin{tabular}{cccccccccccc}
\hline Characteristics & \multicolumn{9}{c}{ RU } & \multicolumn{5}{c}{ RS } \\
\hline Dual diagnosis & $\mathrm{N}$ & Yes & \% of Yes & $\mathrm{N}$ & \% of No & $\mathrm{N}$ & $\mathrm{Y}$ & \% of Yes & $\mathrm{N}$ & $\%$ of No \\
\hline Depression & 20 & 10 & 25.6 & 10 & 25.6 & 19 & 8 & 20.5 & 11 & 28.2 \\
Anxiety & 20 & 7 & 17.9 & 13 & 33.3 & 19 & 6 & 15.4 & 13 & 33.3 \\
PTSD & 20 & 0 & 0.0 & 20 & 51.3 & 19 & 2 & 5.1 & 17 & 43.6 \\
Bipolar & 20 & 2 & 5.1 & 18 & 46.2 & 19 & 5 & 12.8 & 14 & 35.9 \\
Other & 20 & 0 & 0.0 & 20 & 50.0 & 19 & 1 & 2.6 & 18 & 47.4 \\
None & 20 & 7 & 17.9 & 13 & 33.3 & 19 & 3 & 7.7 & 16 & 41.0 \\
Medication & 20 & 12 & 30.8 & 8 & 20.5 & 19 & 15 & 38.5 & 4 & 10.3 \\
\hline
\end{tabular}

\section{Qualitative Themes}

Participants' $(\mathrm{N}=5)$ responses to the identified questions resulted in three general themes: A great need exists around housing services for the population of adult males who complete drug and alcohol treatment and halfway house community reentry services. A second theme is the reported degree of importance for housing and services to accompany transitions back into the community; the third theme is a reported network of alumni from the halfway house, and transitional homes that evolved naturally without facilitation or connection to the treating facility. The final theme is the lack of formal process within the Halfway House for determining what works, what does not work, and who responds to services and who does not. The overarching theme is that a culture of inquirers or interest in the above questions is lacking.

\section{Discussion}

In this study we examined the recovery needs of residentially unstable substance abusers who are provided with transitional housing compared with the recovery needs of substance abusers who are not residentially unstable. Our aim was to consider how these findings might inform efforts to expand service, improve services, and to determine if clients who are in need are provided with a similar level of support from clients who return to housing environments that are more stable and or supportive.

The results suggest that the clients who returned to a residentially stable environment after primary drug and alcohol treatment and halfway house services were delivered were still in need of transitional housing services. Secondly, the need for transitional housing services and other biopsychosocial services is far greater than researchers knew. This mirrors key findings from the literature review which indicated that residential instability and homelessness among addicts are related to a variety of negative recovery outcomes. For example, Callaghan and Cunningham (2002) [2] reveal that "the lack of a fixed address" (p. 405) is a risk factor for re-admission to detoxification. Residential instability, defined as " 1 year or less in the same setting” (Moos et al., 2002, p. 78) [3], is a risk factor for increased drug use symptoms after discharge from in-patient treatment, and homeless addicts achieve better abstinence outcomes when they are provided with housing (McKeown, 2007 [7]; Milby, Schumacher, Wallace, Freedman, \& Vuchinich, 2005 [11]; Anderson, Shannon, Schyb, \& Goldstein, 2002 [8]; Devine \& Wright, 1997 [9]; Stahler \& Cohen, 1995) [10].

\section{Conclusions}

The implications of this study are important to consider in relation to organizational decision to expand housing services; severity levels of clients; and the type of services that should be offered; and the scope of biopsychosocial services that should be provided or collaborated with other community services to meet the needs of this population. The need is so great that a culture may exist that allows the provider to grow the services without insight or knowledge of who needs to be treated; what works for whom; how will other services be provided to meet their needs; and what happens to the individuals who need the service but cannot access the service?

The descriptive quantitative data are consistent with the qualitative feedback. The striking element to the quantitative data appears to be that the determination of which individuals are returning to a stable environment is inaccurate and misleading. The needs of these residentially stable individuals and requirement for additional 
services are just as great as those of the residentially unstable. Secondly, the residentially stable groups are often referred to other transitional services due to the lack of bed space at the facility in this unit of analysis. This particular finding offers guidance into further research that pertains to effectiveness of the service, lack of regulation of the service and the impact of a continuum of care that can improve and reduce the severity levels of this population. The longer individuals with significant biopsychosocial issues remain in a continuum of care, the greater the improvement (White \& Kurtz 2006 [6]; O’Brien \& McLellan, 1996 [5]).

The second consideration from these data must be the reported severity of biopsychosocial issues with depression, anxiety, living wage, employment, lack of transportation and health concerns, which are consistent and significant in the quantitative and qualitative data. This is corroborated in the literature review (O'Brien \& McLellan, 1996) [5].

Lastly, a general finding pertaining to the culture of the organization and the need for the service should be taken to conceptualize residential stability. What does it and what should it mean for this population group? This means that the organization can have a role in developing new services through investments and collaboration within the community. This can result in a clinical and business model that addresses the needs of this population without duplication, and still utilize best practice and evidence based practice to deliver the service (Conahan \& MacIntyre, 2010) [1].

There are limitations to our study that are important to note, the largest of which are its exploratory nature and use of very small, non-representative sample of persons. Findings from our study offer only personal guidelines for other programs that provide substance abuse services, and insight for other programs to consider when addressing the need for housing services for the substance abusing population.

\section{References}

[1] Conahan, J. and MacIntyre, D. (2012) Responding to the Needs of Homeless Substance Abusers. The International Journal of Interdisciplinary Social Sciences, 6, 153-166.

[2] Callaghan, R.C. and Cunningham, J.A. (2002) Gender Differences in Detoxification: Predictors of Completion and ReAdmission. Journal of Substance Abuse Treatment, 23, 399-407. http://dx.doi.org/10.1016/S0740-5472(02)00302-1

[3] Moos, R.H., Nichol, A.C. and Moos, B.S. (2002) Risk Factors for Symptom Exacerbation among Treated Patients with Substance Use Disorders. Addiction, 97, 75-85. http://dx.doi.org/10.1046/j.1360-0443.2002.00063.x

[4] (1999) Client Placement Criteria. http://www.portal.state.pa.us/portal/server.pt/community/pcpc edition 3/21898

[5] O’Brien, C.P. and McLellan, A.T. (1996) Myths about the Treatment of Addiction. The Lancet, 347, 237-240. http://dx.doi.org/10.1016/S0140-6736(96)90409-2

[6] White, W.L. and Kurtz, E. (2006) The Varieties of Recovery Experience: A Primer for Addiction Treatment Professionals and Recovery Advocates. In: White, W.L., Kurtz, E. and Sanders, M., Eds., Recovery Management, Great Lakes Addiction Technology Transfer Center, Chicago. http://asi.nattc.org/regcenters/productdetails.asp?prodID=413\&rcID=3

[7] McKeown, S. (2007) Tackling the Housing Needs of Drug Users: Safer for the User, Safer for the Community. Housing, Care and Support, 10, 23-26. http://dx.doi.org/10.1108/14608790200700011

[8] Anderson, T.L., Shannon, C., Schyb, I. and Goldstein, P. (2002) Welfare Reform and Housing: Assessing the Impact to Substance Abusers. The Journal of Drug Issues, 32, 265-296. http://dx.doi.org/10.1177/002204260203200111

[9] Devine, J.A. and Wright, J.D. (1997) Losing the Housing Game: The Leveling Effects of Substance Abuse. American Journal of Orthopsychiatry, 67, 618-631. http://dx.doi.org/10.1037/h0080259

[10] Stahler, G.J. and Cohen, E. (1995) Homelessness and Substance Abuse in the 1990s. Contemporary Drug Problems, 22, 169-191.

[11] Milby, J.B., Schumacher, J.E., Wallace, D., Freedman, M.J. and Vuchinich, R.E. (2005) To House or Not to House: The Effects of Providing Housing to Homeless Substance Abusers in Treatment. American Journal of Public Health, 95, 1259-1265. http://dx.doi.org/10.2105/AJPH.2004.039743

[12] Bond, J., Kaskutas, L.A. and Weisner, C. (2003) The Persistent Influence of Social Networks and Alcoholics Anonymous on Abstinence. Journal of Studies on Alcohol, 64, 579-588. http://dx.doi.org/10.15288/jsa.2003.64.579

[13] Salomonsen-Sautel, S., Van Leeuwen, J.M., Gilroy, C., Boyle, S., Malberg, D. and Hopfer, C. (2008) Correlates of Substance Use among Homeless Youths in Eight Cities. The American Journal on Addictions, 17, 224-234. http://dx.doi.org/10.1080/10550490802019964

[14] Yunus, M. (2010) Building Social Business. Public Affairs, New York. 
[15] McLellan, A.T., Cacciola, J.C., Alterman, A.I., Rikoon, S.H. and Carise, D. (2006) The Addiction Severity Index at 25 : Origins, Contributions, and Transitions. The American Journal on Addictions, 15, 113-124. http://dx.doi.org/10.1080/10550490500528316

[16] Rubin, A. and Babbie, E.R. (2008) Research Methods for Social Work. Brooks/Cole, Belmont.

[17] Creswell, J.W. and Miller, D.L. (2000) Determining Validity in Qualitative Inquiry. Theory into Practice, 39, 124-130. http://dx.doi.org/10.1207/s15430421tip3903_2

\section{Appendix}

\section{Qualitative Questions}

What were the big issues in your recovery when you arrived for halfway house services? Did you get the supports you needed in each of these areas while you were in treatment at Halfway House? Did the important issues in your recovery change while you were in treatment at the Halfway House?

Where did you go to live when you left the halfway House? Was this living situation supportive of your recovery? Did you get help from anyone in finding a place to live? Who helped, how did they help? If no one helped, how did you find a place to live? Do you think you needed more support in this area? If so, what kind of support?

Is your family supportive of your recovery?

Did you have legal issues when you left? How have these played out since you left the halfway house? Have they affected your sobriety? If so, how? Do you think you needed greater supports in this area during your stay at the Halfway House? Have you had supports in this area since leaving the Halfway House?

Did you have physical health issues when you left? How have these played out since you left? Have they affected your sobriety? If so, how?

Have you been employed since you left? How has your job situation affected your sobriety since discharge? Do you think you needed greater supports in this area during your stay at the Halfway House?

Have you had regular access to a computer and the internet since leaving? If so, where/how? If not, has this affected your recovery?

If the Halfway House were to offer post-discharge services, what services would be most useful to you?

Have you been in contact with others with whom you were in treatment? 